\title{
[gw22-e0318] EXOGENOUS EXPRESSION OF ISL1 GENE IN HUMAN MESENCHYMAL STEM CELLS BY LENTIVIRAL TRANSDUCTION
}

Wang Yinfen', Li Weiqiang², Fang Rong', Liu Jia', Yang Daya', Hu Chengheng ${ }^{11}$ The First Affiliated Hospital Of Sun Yat-sen University, Guangzhou, China; ${ }^{2}$ Center For Stem Cell Biology And Tissue Engineering, Sun Yat-sen University, Guangzhou, China

10.1136/heartjnl-2011-300867.41

Objective To investigate the mRNA and protein expression of Isl1 gene at different time point, which was exogeneously expressed in human mesenchymal stem cells, using lentiviral vector mediated gene transduction technology, and detect expression of downstream gene Nkx2.5 and Flk-1.

Methods 2K7puro/EF1 $\alpha$-Isl1 lentiviral vector was constructed by multi-site gateway technology (promoter EF1 $\alpha$ driving Isl1 gene expression). Lentivirus was produced in virus package cell 293FT cells, and then transducted to human mesenchymal stem cells, afterwards the puromycin was added to the culture medium for screening Is11 positive cells. Post-transduction Isl1 gene expression in positive cells were measured by reverse transcription polymerase chain reaction (RT-PCR) for mRNA over week 1 to week 6 , and western blot for protein over week 1 to week 4 , respectively.

Results Lentiviral vector EF1 $\alpha$-Is11 was successfully constructed, and subsequently transducted hMSCs expressed Isl1, both at mRNA and protein levels. The mRNA expression was shown to be increasing with culture time. The level of expression was significantly greater at week 3 compared to week 1 and week 2 , became stable. In the absence of inductor, the expression of downstream gene Nkx2.5, but not Flk-1, was detected in the hMSCs with Isl1 positive cells.

Conclusion By lentiviral transdution, Isl1 gene was successfully integrated into hMSCs genome with resultant stable expression. Furthermore, the expression of downstream Nkx2.5 gene can be detected in the absence of any inductor, which may have important implications for cardiac differentiation of hMSCs. 